\title{
Sleep related hyper motor epilepsy (SHE): a unique syndrome with heterogeneous genetic etiologies
}

\author{
Francesca Bisulli ${ }^{1,2^{*}}$, Laura Licchetta ${ }^{1,2}$ and Paolo Tinuper ${ }^{1,2}$
}

\begin{abstract}
Sleep-related hypermotor epilepsy (SHE), formerly known as Nocturnal Frontal Lobe Epilepsy is a focal epilepsy characterized by seizures with complex hyperkinetic automatisms and/or asymmetric tonic/dystonic posturing occurring mostly during sleep. SHE is a rare disease with an estimated minimum prevalence of 1.8/100,000 individuals and represent about $10 \%$ of drug-resistant surgical cases. This disorder, though uncommon, is of considerable interest to a broad spectrum of specialists, from child neurologists to neurosurgeons. Distinguishing this condition from nonepileptic paroxysmal behaviour occurring physiologically or pathologically during sleep is often difficult and sometimes impossible on clinical grounds alone, even for experienced epileptologists and sleep physicians. Recognized aetiologies of SHE are heterogeneous and include acquired injuries, genetic causes and structural anomalies such as focal cortical dysplasia. Multiple aetiologies (structural-genetic) are also possible. Non-specific clinical features distinguished different aetiologies even if SHE due to structural lesions usually manifests with early-onset drug-resistant seizures and showed a worse long-term prognosis.

The causative genes for SHE are multiple and encode for proteins involved in different molecular pathways. The cholinergic system and the mTOR pathway are the most relevant. This review will provide an exhaustive overview of the genetic background of SHE.
\end{abstract}

Keywords: Epilepsy, Sleep-related hypermotor epilepsy, Nocturnal frontal lobe epilepsy, Etiology, Genetics; structural-genetic

\section{Sleep-related Hypermotor epilepsy (SHE)}

SHE, formerly Nocturnal Frontal Lobe Epilepsy (NFLE), is a focal epilepsy characterized by hyperkinetic seizures occurring predominantly in clusters during non-REM sleep.

This disorder affects individuals of both sexes and any age, with a peak of seizure onset during childhood and adolescence (Scheffer et al. 1994; Tinuper et al. 2016). A familial form of SHE with autosomal dominant inheritance (ADSHE) has been described. So far, more than 100 families have been identified worldwide (Marini and Guerrini 2007; Steinlein 2014), but no accurate data concerning the prevalence of ADSHE exist.

\footnotetext{
* Correspondence: francesca.bisulli@unibo.it

${ }^{1}$ IRCCS Istituto delle Scienze Neurologiche di Bologna, Bellaria Hospital, Via Altura 3, Bologna, Italy

${ }^{2}$ Department of Biomedical and Neuromotor Sciences (DIBINEM), University of Bologna, Bologna, Italy
}

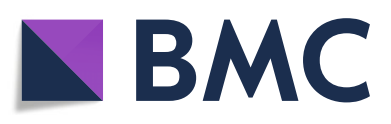

(c) The Author(s). 2019 Open Access This article is distributed under the terms of the Creative Commons Attribution 4.0 International License (http://creativecommons.org/licenses/by/4.0/), which permits unrestricted use, distribution, and reproduction in any medium, provided you give appropriate credit to the original author(s) and the source, provide a link to the Creative Commons license, and indicate if changes were made. The Creative Commons Public Domain Dedication waiver (http://creativecommons.org/publicdomain/zero/1.0/) applies to the data made available in this article, unless otherwise stated. adult population is $1.8-1.9$ per 100,000 (Vignatelli et al. 2015; Vignatelli et al. 2017). However, the disorder is likely to be under diagnosed, or in some cases misdiagnosed. Distinguishing this condition from non-epileptic paroxysmal behaviour occurring physiologically or pathologically during sleep is often difficult and sometimes impossible on clinical grounds alone, even for experienced epileptologists and sleep physicians. As a result, misdiagnosis is common and patients may be denied effective treatments or treated inappropriately, leading to long-term side effects and the social consequences of erroneous epilepsy diagnosis (e.g., impacts on driver's licence).

Most patients show a good response to the pharmacological treatment, low doses of carbamazepine at bedtime being the first choice of therapy. However, about one-third of patients are drug-resistant and only $22 \%$ achieved terminal remission after a median 16-year 
follow-up, most with a remitting pattern from disease onset (Licchetta et al., 2017). These data, showing the poor outcome after a long follow-up, possibly explain the reason why SHE has been reported in up to $10 \%$ of surgical series (Menghi et al. 2018). The surgical outcome seems to be relatively good in this population, especially in patients with positive brain MRI.

\section{From NPD to SHE}

First described in 1981 (Lugaresi and Cirignotta 1981), the condition was initially considered a new motor disorder of sleep, namely parasomnia, and the misleading term Nocturnal Paroxysmal Dystonia (NPD) was introduced. Subsequently, similarity of the attacks to those in patients with frontal lobe epilepsy undergoing neurosurgical evaluation (Williamson et al. 1985; Waterman et al. 1987) and documentation of epileptiform discharges in some patients (Tinuper et al. 1990) proved the epileptic origin of the syndrome. The disorder was therefore re-named Nocturnal Frontal Lobe Epilepsy (NFLE). In the following two decades, the clinical boundaries of the disorder were defined, however many controversial issues highlighted the need to change the nomenclature (Tinuper and Bisulli 2017). In 2014, a Consensus Conference was held in Bologna, Italy (Tinuper et al. 2016). Experts in the field discussed the spectrum of NFLE and produced a final Consensus Statement based on a rigorous protocol addressing nomenclature, electro-clinical definition, diagnostic criteria with levels of certainty supported by available evidence, aetiology, and research needs. The Consensus Conference highlighted three critical issues justifying the change of nomenclature. First, the term nocturnal was considered misleading because it implies a chronobiological pattern of seizure occurrence, whereas evidence indicates that seizure occurrence in sleep is the most important characteristic rather than the time of day. Second, the term frontal lobe is not always appropriate because the characteristic seizures may also arise from extra-frontal areas (Proserpio et al. 2011). Third, the term NFLE did not specify the typical clinical semiology involved, which consists primarily of hyperkinetic seizures (Tinuper et al. 2016). For all these reasons the term Sleep related Hypermotor Epilepsy (SHE) has been proposed to replace NFLE.

\section{Clinical features}

Seizures in SHE are usually brief $(<2 \mathrm{~min}$ in duration), with an abrupt onset and offset and with stereotyped motor patterns. The hypermotor semiology is the primary clinical pattern of the seizures, characterized by hyperkinetic features possibly associated with asymmetric tonic/dystonic posturing with or without head/eye deviation. In a minority of cases the asymmetric dystonic posturing is the unique feature of the seizures (Vignatelli et al. 2015). Seizures typically show variable complexity and duration varying from brief stereotyped sudden arousals from sleep (paroxysmal arousals or minor motor events) to more complex dystonic-dyskinetic seizures and, more rarely, prolonged ambulatory behavior known as "epileptic nocturnal wandering" (Montagna 1992; Provini et al. 1999; Nobili et al. 2003; Terzaghi et al. 2008). Retained awareness during seizures is common and affected individuals may report a distinct aura. Seizures occur typically in cluster during (non-REM) sleep even if episodes during active wakefulness may rarely occur during the patient's lifetime.

Seizure frequency in SHE patients can be very high, ranging from one to more than 50 attacks per night (Scheffer et al. 1994; Provini et al. 1999) and paroxysmal arousals or minor motor events may be even more frequent. SHE patients can complain of nocturnal sleep disruption with spontaneous midsleep awakening, sleep inertia in the morning, tiredness at awakening and excessive sleepiness (Peled and Lavie 1986; Maccario and Lustman 1990; Schwalen and Jorg 1998; Zucconi et al. 2000; Alanis-Guevara et al. 2005) impacting negatively on their quality of life. Although daytime sleepinessrelated symptoms could be common in SHE patients, the frequency of excessive daytime sleepiness did not statistically differ compared to controls (Vignatelli et al. 2006).

\section{Neurophysiological features}

Background activity is usually normal. Interictal EEG is normal in about half cases (Licchetta et al. 2017) or may demonstrate rare epileptiform abnormalities, enhanced by sleep deprivation and occurring mainly during sleep (Menghi et al. 2018). The ictal scalp EEG may be normal or may only demonstrate movement artifacts. Epileptiform abnormalities, rhythmic slow activity or diffuse background flattening over frontal areas are seen in 50$60 \%$ of cases (Licchetta et al. 2017).

Scalp EEG and invasive intracranial stereoelectroencephalography recordings (SEEG) documented a frontal lobe origin of seizures in most cases (Nobili et al. 2007; Rheims et al. 2008). However, in up to $20 \%$ of drug resistant cases the ictal discharges may arise from various extra-frontal areas including temporal (Nobili et al. 2004; Vaugier et al. 2009), insulo-opercular (Ryvlin et al. 2006; Dobesberger et al. 2008; Nguyen et al. 2009; Proserpio et al. 2011) and parietal (Montavont et al. 2013; Gibbs et al. 2016) cortices, then propagating to the frontal cortex and resulting in hypermotor seizures.

\section{Diagnostic criteria}

The clinical history and clinicffttal semiology of the attacks are the main criteria to establish the diagnosis, as both interictal and ictal EEG may be uninformative 
(Scheffer et al. 1994; Oldani et al. 1998; Nobili et al. 2007; Licchetta et al. 2017). Three categories for the diagnosis with different levels of certainty have been proposed: 1) Witnessed (possible), based on the description of the core clinical features, as provided by an eyewitness; 2) Video Documented (clinical), based on the evaluation of at least 1 entire (preferably 2 ) video recorded hyperkinetic episode, confirmed to be typical by witness; 3) Video-EEG documented (confirmed), requiring the video-polygraphic recording of stereotyped events (one or two) and ictal or interictal epileptiform abnormalities (Tinuper et al. 2016).

\section{Aetiology}

Etiology is unknown in the majority of patients. Recognized etiologies of SHE are heterogeneous and include acquired injuries, genetic causes and structural anomalies such as focal cortical dysplasia (FCD). Multiple etiologies (structural-genetic) are also possible. Non-specific clinical features distinguished different etiologies (Tinuper et al. 2016) even if SHE due to structural lesions (FCD) usually manifests with early-onset drug-resistant seizures (Nobili et al. 2009) and showed a worse long-term prognosis (Licchetta et al. 2017). In these cases, epilepsy surgery and removal of the epileptogenic zone could represent a highly effective treatment option (Nobili et al. 2007).

Most patients (86\%) are sporadic cases, while $14 \%$ reported a family history for epilepsy with only $5 \%$ of cases showing a clear-cut autosomal dominant pattern of inheritance, i.e. ADSHE (Licchetta et al. 2017). So far ADSHE has been associated with mutations in several genes, encoding proteins involved in different biological pathways. Although the diverse underlying aetiologies and networks involved in its pathogenesis, according to the current state of knowledge, it is considered a single syndrome, defined by clinical manifestations (i.e. hypermotor seizures) resulting from presumed shared downstream mechanisms occurring during sleep/wake oscillation changes. In clinical practice it is most helpful to consider SHE as a single syndrome because it requires a specific diagnostic work-up and therapeutic approach e (Tinuper et al. 2016).

\section{Genetics SHE}

SHE is the first epilepsy syndrome in which a genetic aetiology was documented. It is also the first the first epilepsy channelopathy described, as it was initially related to mutations in genes coding for subunits of the neuronal nicotinic acetylcholine receptor (nAChR), CHRNA4, CHRNB2 and CHRNA2 (Steinlein et al. 1995).

Subsequently, SHE has been associated with mutations in several other genes, encoding proteins involved in different biological pathways, such as, CRH, KCNT1, DEPDC5, NPRL2, NPRL3 and PRIMA1.
Inherited SHE usually shows an autosomal dominant pattern of transmission, except for a single reported family mutated in PRIMA1, showing an autosomal recessive inheritance (Hildebrand et al. 2015).

\section{Ach receptor genes}

In 1994, Scheffer et al. (Scheffer et al. 1994) described the first large Australian family with SHE inherited in an autosomal dominant manner and named this condition Autosomal Dominant Nocturnal Frontal Lobe Epilepsy (ADNFLE). In this family, SHE had been misdiagnosed as a sleep disorder in many affected members.

Marked variations in severity have been observed amongst different members. Further molecular genetic studies established linkage to chromosome 20q13.2q13.13 in some families (Phillips et al. 1995), leading to the subsequent identification of the causative role of the gene coding for the $\alpha$ 4-subunit of the nAChR, CHRNA4 (Cholinergic Receptor Nicotinic Alpha 4 Subunit, MIM *118504) (Steinlein et al. 1995). Following the initial recognition, more than a hundred families have been described worldwide (Marini and Guerrini 2007) and mutations in two homologous genes, CHRNB2 (Cholinergic Receptor Nicotinic Beta 2 Subunit, MIM *118507) and CHRNA2 (Cholinergic Receptor Nicotinic Alpha 2 Subunit, MIM *118502), encoding the $\beta 2$ and $\alpha 2$ subunit of the nAChR respectively, have been identified (De Fusco et al. 2000; Aridon et al. 2006). The phenotype produced by mutations of the three nAChR subunit genes is generally indistinguishable (McLellan et al. 2003).

By now, 14 different mutations in CHRNA4, CHRNB2 and CHRNA2 have been reported in 20 ADSHE pedigrees and three sporadic cases, as reported in Table 1. Overall, they account for less than 20\% of SHE/ADSHE cases, reflecting the genetic heterogeneity of the syndrome and the possible role of systems other than the cholinergic one, involved in its pathogenesis (Steinlein et al. 2012).

In-vitro analyses of the functional properties of $\mathrm{nAChR}$ disclosed a functional gain (i.e. an increase in acetylcholine sensitivity) (Bertrand et al. 2002) of mutant receptors associated with ADSHE that may underlie the neuronal network dysfunction responsible for the epileptic seizures. Positron-emission tomography (PET) studies in ADSHE patients suggest hyperactivation of the cholinergic pathway ascending from the brainstem (Picard et al. 2006). Cholinergic neurons modulate sleep and arousal at both thalamic and cortical levels and their involvement in sleeprelated disorders is plausible, although the pathophysiological mechanism remains elusive.

Interestingly, a high prevalence of NREM arousal parasomnias has been reported not only by SHE probands but also by their healthy relatives (Bisulli et al. 2010; 
Table 1 Families and sporadic cases with mutations in CHRNA4, CHRNB2 and CHRNA2 reported so far

\begin{tabular}{|c|c|c|c|c|}
\hline \multicolumn{2}{|c|}{ MUTATIONS } & \multicolumn{3}{|c|}{ FAMILIES REPORTED } \\
\hline GENE & AA CHANGE/DOMAIN & $\mathrm{N}$ & ORIGIN & REFERENCES \\
\hline \multirow[t]{13}{*}{ CHRNA4 } & $\mathrm{S} 248 \mathrm{~F} / \mathrm{TM} 2$ & 4 & British-AU & Steinlein et al. 1995 \\
\hline & & & Spanish & Sáenz et al. 1999 \\
\hline & & & Norwegian & Steinlein et al. 2000 \\
\hline & & & Scottish & McLellan et al. 2003 \\
\hline & S252 L/ TM2 & 4 & Japanese & Hirose et al. 1999 \\
\hline & & & Lebanese & Phillips et al. 2000 \\
\hline & & & Korean & Cho et al. 2003 \\
\hline & & & Polish & Rozycka et al. 2003 \\
\hline & & 1 & Italian (S) & Sansoni et al. 2012 \\
\hline & 776ins3/ TM2 & 1 & Norwegian & Steinlein et al. 1997 \\
\hline & T265I/ TM2 & 1 & German & Leniger et al. 2003 \\
\hline & R336H/ Intracel loop 2 & 1 & Chinese & Chen et al. 2009 \\
\hline & c. $823 \mathrm{~A}>\mathrm{T}$ & 1 & Chinese (S) & Wang et al. 2014 \\
\hline \multirow[t]{9}{*}{ CHRNB2 } & V287 L/ TM2 & 1 & Italian & De Fusco et al. 2000 \\
\hline & V287 M/ TM2 & 2 & Scottish & Phillips et al. 2001 \\
\hline & & & Spanish & Díaz-Otero et al. 2008 \\
\hline & L301 V/ TM3 & 1 & Turkish Cypriot & Hoda et al. 2008 \\
\hline & V308A/ TM3 & 2 & Scottish & Hoda et al. 2008 \\
\hline & & & English & \\
\hline & I312M/ TM3 & 2 & English & Bertrand et al. 2005 \\
\hline & & & Korean & Cho et al. 2008 \\
\hline & V337G/ TM3-intrac loop & 1 & Chinse (S) & Liu et al. 2011 \\
\hline \multirow[t]{2}{*}{ CHRNA2 } & I279N/TM1 & 1 & Italian & Aridon et al. 2006 \\
\hline & 1297F/TM2 & 1 & Italian & Conti et al. 2015 \\
\hline
\end{tabular}

Licchetta et al. 2017) suggesting a possible common background. The relationship between these disorders is also supported by the neurophysiological and neuroimaging evidence that the pathway controlling physiologic arousal is impaired in both conditions (Picard et al. 2006; Montagna et al. 2008; Fedi et al. 2008).

Seizure frequency improved in a single patient with refractory ADSHE after nicotine transdermal patches treatment (Willoughby et al. 2003).

The favourable effect of nicotine on seizure frequency was also described in 9 out of 22 patients from two European ADSHE families carrying CHRNA4 mutations (Brodtkorb and Picard 2006). Considering the role of the cholinergic system in arousal regulatory processes, these observations suggested a possible link between nicotine defect, alteration of arousal regulation and seizures in SHE/ADSHE patients. However, despite the reported positive effect of nicotine in reducing seizure frequency, a case-control family study, did not find a higher tendency to smoke tobacco in SHE patients and their relatives compared to the control cases (Naldi et al. 2013).
Recently, Puligheddu and colleagues have shown that fenofibrate, an agonist at peroxisome proliferatoractivated receptor alpha (PPARa) acting as a negative modulator of nAChRs, may have beneficial effects both in mutated mouse models of SHE and pharmacoresistant SHE patients (Puligheddu et al. 2017). Interestingly, good results with fenofibrate administration have been obtained both in the mutated (CHRNA2 and CHRNA4) and non-mutated SHE patients.

\section{CRH}

Other mutations besides the ones coding for the nAChR subunits have been reported in both sporadic and ADSHE cases. In 2005, Combi et al. found two new nucleotide variations in the $C R H$ (corticotropin-releasing hormone, MIM *122560) promoter in ADSHE pedigrees and sporadic cases (Combi et al. 2005) but these findings were not replicated in larger series. The first one (g.1470G > A) recurred in three ADSHE pedigrees and two patients without family history and it was shown to increase $\mathrm{CRH}$ levels. The same change was later 
identified in two affected siblings of another family, but it was also present in a homozygous status in the healthy father (Combi et al. 2008), thus reducing the likelihood that the change is pathogenic. The second one (g.1166G > C) was found only in the index-case of a family (Combi et al. 2005) and later recognized as noncausative (Combi et al. 2008).

Finally, a novel heterozygous exonic missense change was detected in an additional ADSHE family. In vitro assay in this case showed decreased CRH concentrations (Sansoni et al. 2013).

$C R H$ encodes for a neurotransmitter/neuromodulator widely distributed throughout the central nervous system that acts in extrahypothalamic circuits to integrate a multisystem response to stress that controls numerous behaviours such as sleep and arousal (Combi et al. 2005) - The authors suggested that altered (decreased/increased) CHR levels cause increased susceptibility of seizures through excessive sleep fragmentation and brain hyperexcitability (Combi et al. 2005).

\section{KCNT1}

Further insight into the genetic background of SHE occurred only from 2012 when, combining genome-wide linkage analysis with novel Next Generation Sequencing (NGS) techniques, Heron and coauthors identified a novel gene for SHE, KCNT1 (Potassium Sodium-Activated Channel Subfamily T Member 1, MIM *608167), encoding a subunit of the sodium-activated potassium channel (Heron et al. 2012). Mutations in KCNT1 were detected in three ADSHE families with complete penetrance and a sporadic case, all with early-onset refractory seizures, possible intellectual disability and psychiatric or behavioral problems including psychosis, catatonia and aggression (Heron et al. 2012). Co-occurrence of mild malformation of cortical development (MCD), namely histologicallyconfirmed FCD type I and periventricular nodular heterotopia, have been reported very recently in some individuals (Rubboli et al. 2018).

Simultaneously, de novo gain-of-function mutations in KCNT1were identified in six out of 12 unrelated individuals with Malignant Migrating Focal Seizures of Infancy (MMFSI) (Barcia et al. 2012), a rare early onset epileptic encephalopathy characterized by refractory, polymorphous focal seizures and arrest of psychomotor development within the first 6 months of life (Coppola et al. 1995).

KCNT1 is expressed in the neurons of the frontal cortex (Bhattacharjee et al. 2002) and assemble with KCNT2 to form heterotetrameric channel complexes composed of a small amino-terminal domain, a transmembrane domain containing six segments and a large intracellular carboxy-terminal domain containing tandem regulators of potassium conductance domains and an NAD + binding domain. Its activity contributes to the slow hyperpolarization that follows repetitive firing, regulates the rate of bursting and enhances the accuracy with which action potentials lock incoming stimuli (Bhattacharjee and Kaczmarek 2005; Brown et al. 2008). All the mutations initially described in both ADSHE and MMFSI were clustered around the regulator of potassium conductance and Nicotinamide Adenine Dinucleotide (NAD+) binding domains, which also interacts with a protein network, including fragile $\mathrm{X}$ mental retardation protein. Functional study documented that KCNT1 mutations cause a constitutive hyperactivation of the channel that impairs its gating and suppress its subconductance states whit effect on ion currents and increased amplitude. Moreover, they may also alter the conformation of the C-terminal region and its ability to interact with developmentally relevant proteins (Barcia et al. 2012). ADSHE mutations were associated with currents approximately 3-fold larger than wild type, while those associated with MMFSI were about 5-fold greater. These differences in the increased ion current amplitude seemed to explain the diversity on phenotypes associated with KCNT1 mutations (Milligan et al. 2014). Some of the variants recurred in several patients, suggesting the presence of mutational "hot spots" in KCNT1 (Møller et al. 2015). Specific mutations (p.G288S and p.R398Q) can lead to either ADSHE or MMFSI, even within the same family, indicating that genotype-phenotype correlations are not straightforward (Kim et al. 2014; Møller et al. 2015).

In the last few years, $K C N T 1$ has been implicated in a wide spectrum of focal/multifocal epilepsies and early onset epileptic encephalopathies, in addition to ADSHE and MMFSI phenotypes (Shimada et al. 2014; Møller et al. 2015; Ohba et al. 2015; Rizzo et al. 2016).

Very recently, a new therapeutic approach with quinidine was tested in few drug-resistant epileptic patients carrying KCNT1 genetic mutations, no one fitting SHE phenotype, with conflicting results (Mikati et al. 2015; Abdelnoura et al. 2018).

Surgical treatment of the three unrelated patients with FCD type I reported was ineffective in two individuals (Engel Class IV) and only ameliorate seizure frequency in one (Engel Class II). The authors postulated that the poor surgical outcome may be due to the diffuse nature of FCD type I or to a wider epileptogenic network caused by germline KCNT1 mutations that sustains seizure propensity after epilepsy surgery (Rubboli et al. 2018).

\section{GATOR1-complex genes}

In 2013, mutations in DEPDC5 (DEP Domain Containing 5, MIM *614191) were implicated in familial focal epilepsy with variable foci (FFEVF) (Dibbens et al. 2013), as well as and in a variable percentage $(12.5-37 \%)$ of 
heterogenous familial FEs, including ADSHE (Ishida et al. 2013; Picard et al. 2014).

In particular, DEPDC5 loss-of-function mutations were found in the $13 \%$ of a series of 30 families with ADSHE presentation (Picard et al. 2014). Electro-Clinical assessments revealed a higher rate of drug resistance and of daytime seizures compared to classical phenotype (Picard et al. 2014). DEPDC5 mutations are associated with both lesional and non-lesional epilepsies, even within the same family. In some individuals, brain MRI disclosed MCD, ranging from FCD to subtle band heterotopia, with the predominant pattern being bottom-of-the-sulcus dysplasia, a variety of FCD type IIb (Scheffer et al. 2014) (Fig. 1ac; Fig. 2a-c).

Subsequently, mutations in NPRL2 (NPR2-like Protein, MIM "607072) (Ricos et al. 2016), NPRL3 (Nitrogen Permease Regulator-like 3, MIM *600928) (Korenke et al. 2016) have been reported in ADSHE and sporadic cases.
DEPDC5, NPRL2 and NPRL3 are components of the GATOR1 complex (Gap Activity TOward Rags 1), a negative regulator of the mammalian target of rapamycin (mTOR) complex1 (mTORC1) (Bar-Peled et al. 2013). Most of the variants described in these genes are loss of function mutations, with impact on the protein product and consequent hyperactivation of mTORC1 pathway (van Kranenburg et al. 2015). In line with this evidence, so far a wide number of germline and somatic (brain-only) mutations of the mTOR-pathway genes have been associated with a range of lesional and non-lesional FEs. With regards to lesional cases, mutations in these genes have been identified in several MCDs and in particular in up to $46 \%$ of FCD type IIb (Nakashima et al. 2015; Scheffer et al. 2014; Lal et al. 2014; Martin et al. 2014; Scerri et al. 2015; D'Gama et al. 2015; Baulac et al. 2015).

Additional insights into the role of DEDCD5 in FCDrelated focal epilepsies derive from the rat model (Marsan
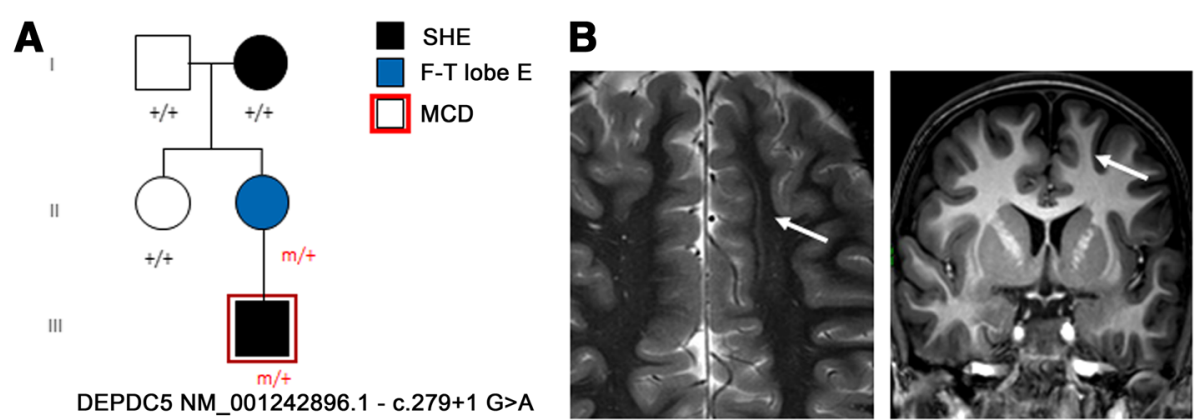

C

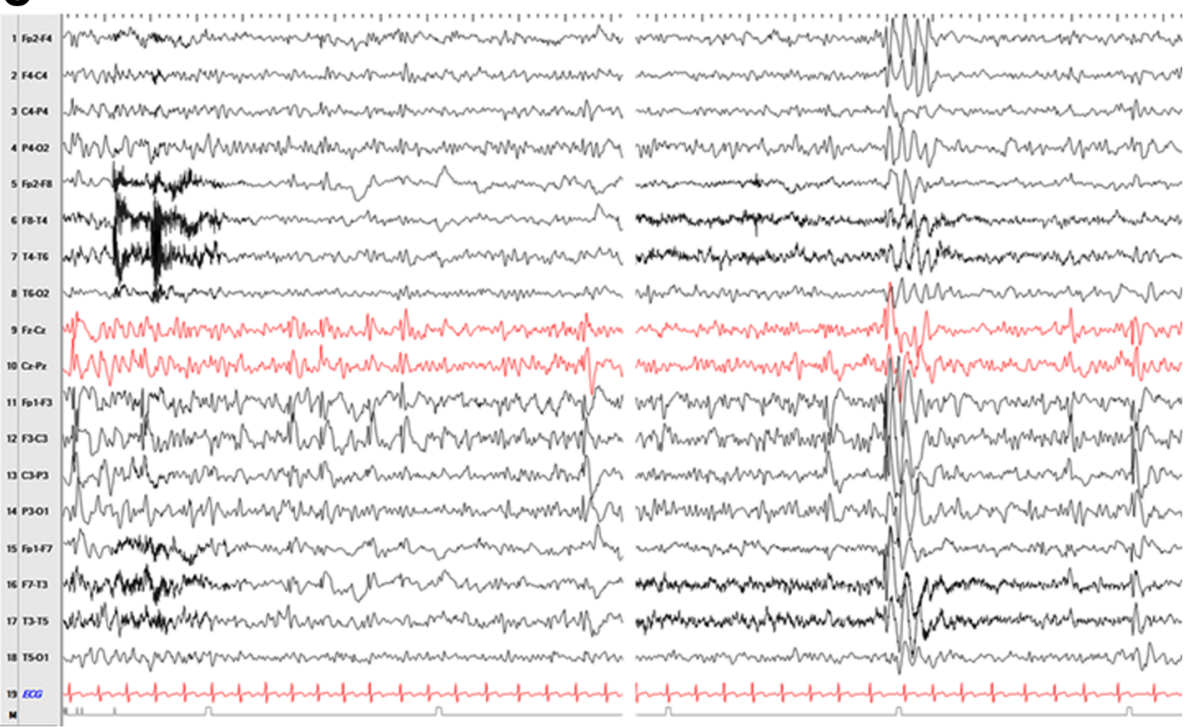

Fig. 1 a Pedigree of the family carring the splicing mutation in DEPDC5 c.279 + $1 \mathrm{G}>$ A. Individual III.1 showed Malformation of cortical development at $3 \mathrm{~T}$ brain MRI. Previously published in Dibbens et al. 2013 (fam I); Scheffer et al. 2014 (fam C). b Brain MRI of individual III.1. The white arrows point to the unilateral subtle band heterotopia within the white matter adjacent to dysplastic cortex in the left frontal lobe. Blurring of the gray-white matter junction involving part of the cingulate cortex and left frontal cortex was seen. $\mathbf{c}$ Interictal EEG of the same individual (III.1) showing frequent spike-wave discharges over the left frontal region (sometimes with phase opposition on F3) enhanced by drowsiness and light sleep, spreading to the ipsilateral and contralateral hemispheres 


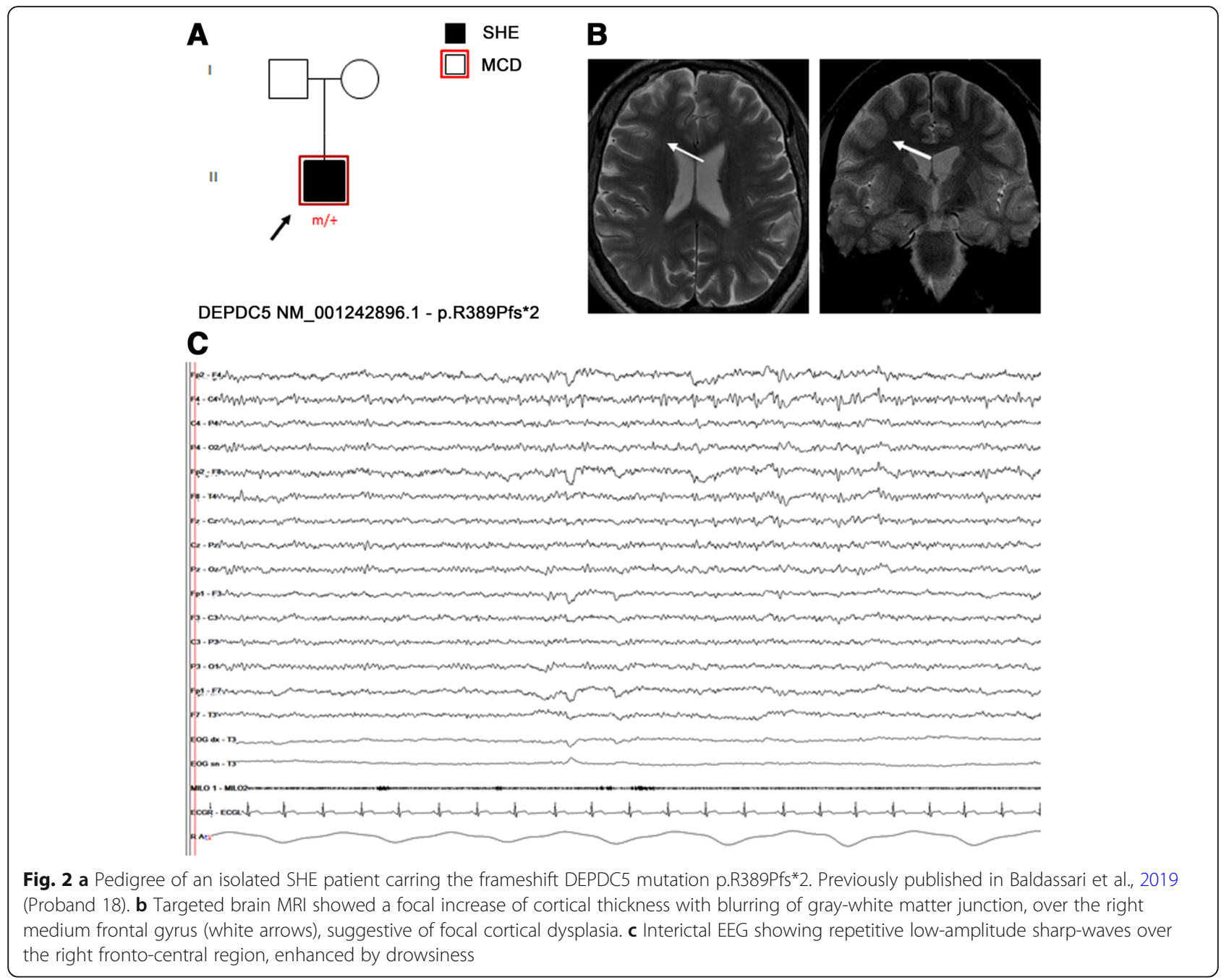

et al. 2016). The heterozygous rats exhibited an altered cortical neuron excitability and firing patterns and cortical cytomegalic dysmorphic neurons and balloon-like cells strongly expressing phosphorylated rpS6, supporting mTORC1 upregulation. These neuropathological abnormalities are reminiscent of the hallmark brain pathology of human FCD.

These data showed that genetic and structural cause are not mutually exclusive either and, in particular, SHE related to GATOR1-complex genes may have a geneticstructural etiology.

FCDs represent the most common, potentially treatable architectural disorder underlying FE, responsible for up to $42 \%$ of drug-resistant cases (Harvey et al. 2008). In general, epilepsy surgery is a highly effective curative option in these patients, affording the opportunity to achieve seizure freedom and potential medication withdrawal also with improvements in quality of life, employment rates and school attendance (Wiebe et al. 2001).
Although mutated patients who have undergone epilepsy surgery are anecdotal, surgery has proved to be curative in cases with MCD clearly detectable with conventional neuroimaging (Baulac et al. 2015), suggesting that epileptogenesis is underpinned by a genetically determined, focal cerebral structural lesion, even in the presence of germline mutations. On the other hand the role of surgery in non lesion cases with germline mutation is still controversial/needs to be clarify. In the cited series non lesional cases who underwent surgery had a worse outcome compared with the lesional ones (Baulac et al. 2015). Moreover, a SEEG study in a patient carrying DEPDC5 truncating mutation failed to identify a definitive epileptogenic zone (Fig. 3a-c). These evidences suggest that DEPDC5 germline mutations could play a role in non-lesional, refractory focal epilepsies with multiple independent epileptogenic foci or widespread epileptogenic networks (Ferri et al. 2017). According to this hypothesis the presence of germinal mutations in mTOR 

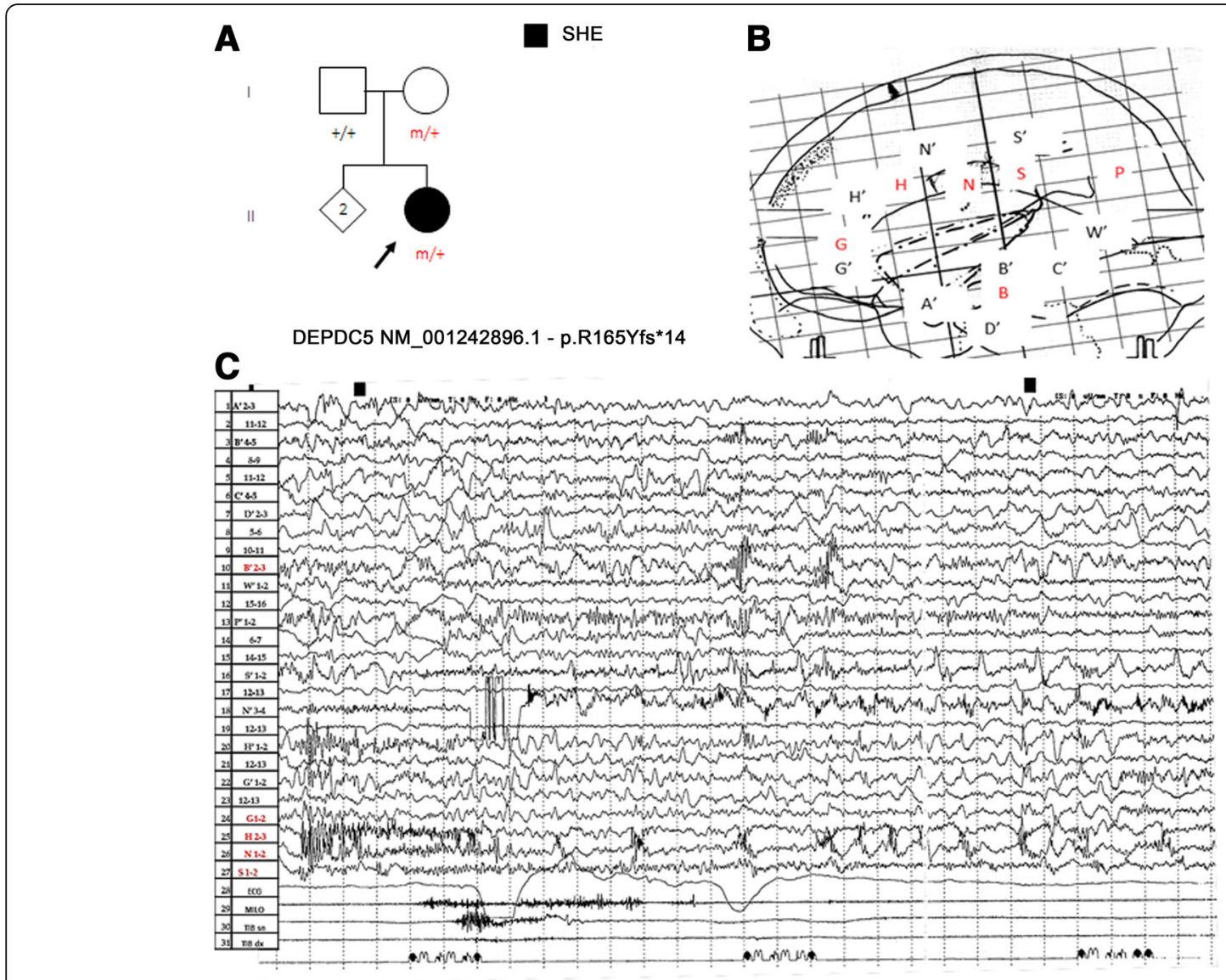

B

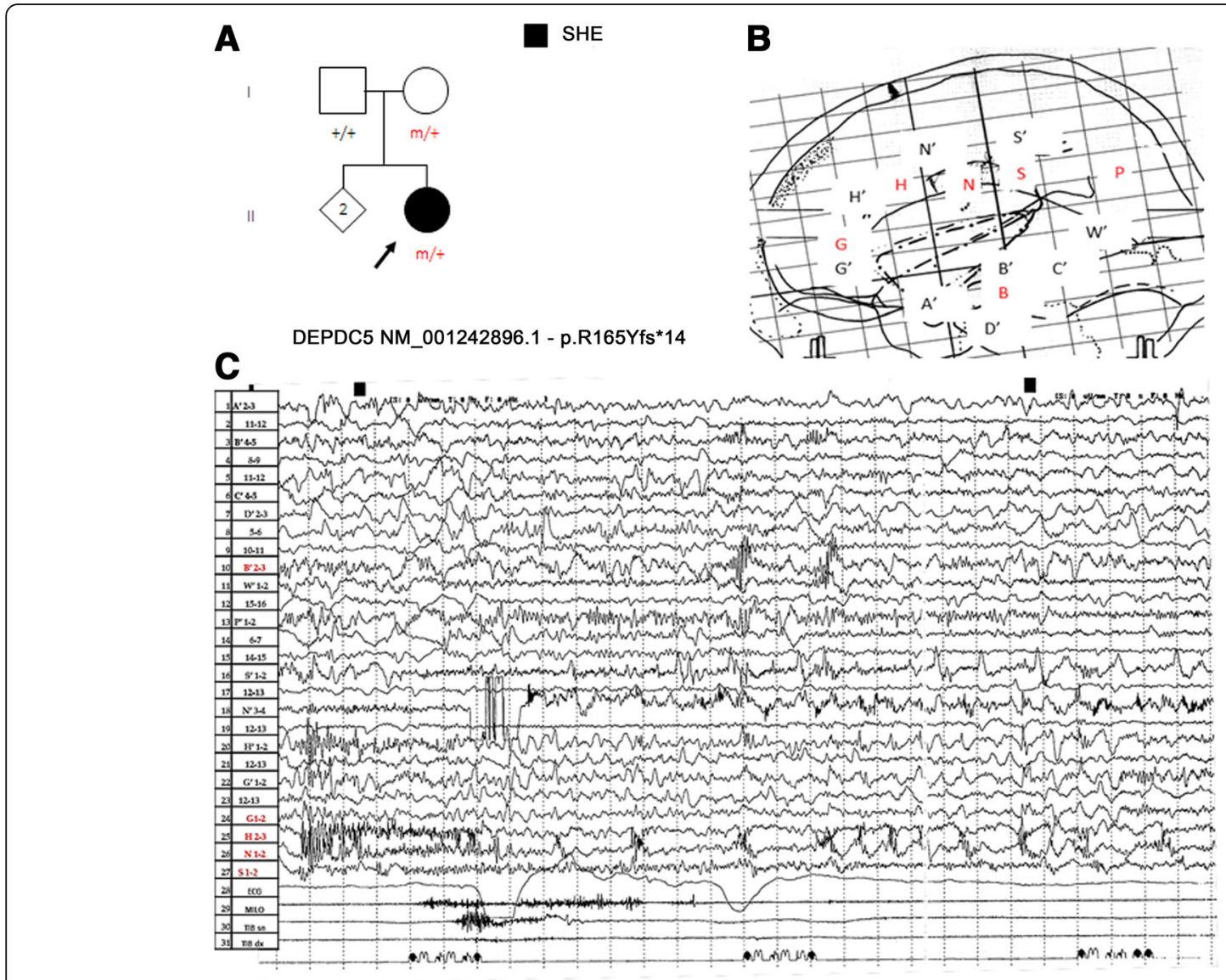

Fig. 3 a Pedigree of a 57-year-old female with a frameshift mutation of DEPDC5, p.Arg165Tyrf**14 inherited by her healthy mother. Previously published in Ferri et al. 2017. b Stereotactic scheme of the patient who at age 43 years underwent stereoelectroencephalography (SEEG) study with bilateral limbic exploration extended to the inferior parietal lobe. The SEEG exploration shown (lateral view) included 15 intra-cerebral electrodes implanted mainly on the left. The EEG focus area was mainly explored by electrode $H$. Black letters with the accent $\left(A^{\prime}, B^{\prime}, C^{\prime}, D^{\prime}, G^{\prime}, H^{\prime}\right.$, $\left.N^{\prime}, S^{\prime}, W^{\prime}\right)$ indicates left side; red letters $(B, G, H, N, S, P)$ indicated the right. c SEEG ictal recording showing fast polyspike activity over both the anterior-mid cingulate gyrus preceding a typical nocturnal hypermotor seizure, prevailing on right central-anterior cingulate cortex ( $\mathrm{H}$ electrode). Note that interictal activity is recorded also in electrodes remote from ictal onset zone. Although the electrical pattern was suggestive for focal cortical dysplasia, tailored brain MRI was unrevealing

genes could represent a contraindication not only for surgery but possibly for presurgical invasive procedures (i.e. stereoEEG, corticography). However, given the association of DEPDC5 mutations also with FCD type I lesions (Baulac et al. 2015), in these non lesional cases it cannot be excluded the presence of multiple, diffuse subtle dysplastic areas missed by conventional brain MRI.

\section{CABP4}

A novel missense mutation in the CABP4 gene encoding the neuronal $\mathrm{Ca} 2+$-binding protein $4(\mathrm{CaBP} 4)$ has been found in a Chinese family including 11 individuals diagnosed with ADSHE (Chen et al. 2017).

\section{PRIMA1}

In a two-generation Australian family of Italian origin affected with SHE and ID, Hildebrand and coauthors identified by Whole Exome Sequencing (WES) analysis a homozygous mutation in PRIMA1 (Hildebrand et al. 2015). This gene encodes a transmembrane protein that anchors acetylcholinesterase (AChE), the enzyme hydrolyzing Ach to membrane rafts of neurons. The c. $93+2 \mathrm{~T}>\mathrm{C}$ mutation identified leads to knockout of PRIMA1, with reduction of AChE and accumulation of acetylcholine at the synapse, as shown in PRIMA1 knockout mice. The authors concluded that, similarly to the gain of function mutations of the genes coding for nAChR subunits, the enhanced cholinergic responses are the likely cause of severe SHE and intellectual disability in this family. However, apart from this single pedigree, this finding has not been replicated yet, as no other mutations were identified in a confirmation cohort of hundreds of SHE probands (Hildebrand et al. 2015). 


\section{Conclusions}

SHE is a heterogeneous genetic syndrome, caused by genes involved in different molecular pathways. Despite a great effort to study the genetic background of SHE, a genetic cause may be recognizable in a very low percentage of sporadic cases and in less than 30\% of ADSHE families, with incomplete penetrance (Kurahashi $\mathrm{H}$ et al., 2002). This is a cumulative estimate coming from studies of different case-series (principally families), each focused on the screening for mutations in a singular SHE gene. A systematic genetic characterization of a population of familial and sporadic patients diagnosed with SHE based on reliable diagnostic criteria is lacking.

Moreover, to date, there are no clear-cut correlations between disease severity, genetic findings and functional effects of the known genetic mutations (Tinuper et al. 2016) and further studies focused on genotypephenotype correlations in SHE are needed. By now, KCNT1 gene mutated SHE patients seem to present a more severe form (Heron et al. 2012), whereas mutations in GATOR1-complex genes have been implicated in genetic-structural etiology of SHE.

Although at present genetic testing has limited usefulness in clinical practice, as genetics explain a minority of SHE patients, from a clinical perspective, analysis of SHE-related genes is worth even in isolated cases for whom a genetic aetiology is not primarily considered, because of possible implications for the diagnostic workup and clinical management. In particular, detection of mutations in GATOR1-complex genes represent a red flag for FCDs, the most common potentially treatable architectural disorder underlying refractory FE. In nonlesional cases carrying pathogenic variants of GATOR1complex genes, repeated and careful review of targeted, high-resolution imaging is needed to highlight subtle structural abnormalities susceptible of surgery. Moreover, the identification of pathogenic or possibly pathogenic mutations in GATOR1-complex genes, as well as in genes coding proteins acting upstream in the mTOR pathway could have important future therapeutic implications even in mutated patients considered not eligible for surgery. In fact, the development of a novel class of therapies based on mTOR inhibitors, whose prototype is rapamycin, will improve the treatment and prognosis of these patients. Both preclinical and clinical trials using mTOR inhibitors to treat epilepsy, and possibly prevent it, are currently underway (Citraro et al. 2016).

\footnotetext{
Abbreviations

AChE: Acetylcholinesterase; ADNFLE: Autosomal dominant nocturnal frontal lobe epilepsy; ADSHE: Autosomal dominant sleep-related hypermotor epilepsy; FCD: Focal cortical dysplasia; FE: Focal epilepsy; FFEVF: familial focal epilepsy with variable foci; mTOR: mammalian Target Of Rapamycin; mTORC1: mammalian Target Of Rapamycin Complex1; nAChR: neuronal nicotinic ACetylcholine Receptor; NAD: Nicotinamide adenine dinucleotide; NFLE: Nocturnal frontal lobe epilepsy; NPD: Nocturnal paroxysmal dystonia;
}

PET: Positron-emission tomography; PPARa: Peroxisome proliferator-activated receptor alpha; SEEG: Stereoelectroencephalography; SHE: Sleep-related hypermotor epilepsy; WES: Whole exome sequencing

\section{Acknowledgements}

We would like to acknowledge all our collaborators of Bellaria Hospital, Bologna, especially Dr. Federica Pondrelli and Dr. Toni Francesco. We are also grateful to all the EEG technicians of our staff, in particular Francesco Mignani.

\section{Authors' contributions}

BF drafted the work and substantively revised it; LL drafted the work; TP revised the work. All authors read and approved the final manuscript.

\section{Funding}

None.

\section{Availability of data and materials}

Not applicable.

\section{Ethics approval and consent to participate}

Not applicable.

\section{Consent for publication \\ Not applicable.}

\section{Competing interests}

The authors declare that they have no competing interests.

Received: 25 March 2019 Accepted: 24 June 2019

Published online: 17 July 2019

\section{References}

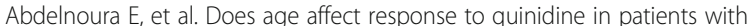
KCNT1 mutations? Report of three new cases and review of the literature. Seizure. 2018;55:1-3.

Alanis-Guevara I, et al. Sleep disturbances, socioeconomic status, and seizure control as main predictors of quality of life in epilepsy. Epilepsy Behav. 2005; 7:481-5.

Aridon $\mathrm{P}$, et al. Increased sensitivity of the neuronal nicotinic receptor alpha 2 subunit causes familial epilepsy with nocturnal wandering and ictal fear. Am J Hum Genet. 2006;79:342-50.

Baldassari S, et al. The landscape of epilepsy-related GATOR1 variants. Genet Med. 2019;21(2):398-408

Barcia G, et al. De novo gain-of-function KCNT1 channel mutations cause malignant migrating partial seizures of infancy. Nat Genet. 2012;44(11):1255-9.

Bar-Peled $L$, et al. A tumor suppressor complex with GAP activity for the rag GTPases that signal amino acid sufficiency to mTORC1. Science. 2013;340: 1100-6.

Baulac S, et al. Familial focal epilepsy with focal cortical dysplasia due to DEPDC5 mutations. Ann Neurol. 2015;77:675-83.

Bertrand D, et al. How mutations in the nAChRs can cause ADNFLE epilepsy. Epilepsia. 2002;43(Suppl 5):112-22.

Bertrand D, et al. The CHRNB2 mutation 1312M is associated with epilepsy and distinct memory deficits. Neurobiol Dis. 2005;20(3):799-804.

Bhattacharjee A, Kaczmarek LKJ. For K+ channels, $\mathrm{Na}+$ is the new Ca2+. Trends Neurosci. 2005;28:422-8.

Bhattacharjee $\mathrm{A}$, et al. Localization of the Slack potassium channel in the rat central nervous system. J Comp Neurol. 2002;454:241-54.

Bisulli $F$, et al. Increased frequency of arousal parasomnias in families with nocturnal frontal lobe epilepsy: a common mechanism? Epilepsia. 2010;51: 1852-60.

Brodtkorb E, Picard F. Tobacco habits modulate autosomal dominant nocturnal frontal lobe epilepsy. Epilepsy Behav. 2006;9:515-20.

Brown MR, et al. Amino-termini isoforms of the slack $\mathrm{K}+$ channel, regulated by alternative promoters, differentially modulate rhythmic firing and adaptation. J Physiol Lond. 2008:586:5161-79.

Chen $Y$, et al. A novel mutation of the nicotinic acetylcholine receptor gene CHRNA4 in sporadic nocturnal frontal lobe epilepsy. Epilepsy Res. 2009;83(23):152-6. 
Chen $\mathrm{ZH}$, et al. Exome sequencing identified a novel missense mutation c. 464G >a (p.G155D) in Ca2+-binding protein 4 (CABP4) in a Chinese pedigree with autosomal dominant nocturnal frontal lobe epilepsy. Oncotarget. 2017; 8:78940-7.

Cho YW, et al. A Korean kindred with autosomal dominant nocturnal frontal lobe epilepsy and mental retardation. Arch Neurol. 2003;60:1625-32.

Cho YW, et al. Autosomal dominant nocturnal frontal lobe epilepsy and mild memory impairment associated with CHRNB2 mutation 1312M in the neuronal nicotinic acetylcholine receptor. Epilepsy Behav. 2008;13:361-5.

Citraro R, et al. mTOR pathway inhibition as a new therapeutic strategy in epilepsy and epileptogenesis. Pharmacol Res. 2016;107:333-43.

Combi R, et al. Two new putative susceptibility loci for ADNFLE. Brain Res. 2005; 67:257-63.

Combi R, et al. Compound heterozygosity with dominance in the Corticotropin releasing hormone $(\mathrm{CRH})$ promoter in a case of nocturnal frontal lobe epilepsy. J Sleep Res. 2008;17(3):361-2.

Conti $V$, et al. Nocturnal frontal lobe epilepsy with paroxysmal arousals due to CHRNA2 loss of function. Neurology. 2015;84(15):1520-8.

Coppola G, et al. Migrating partial seizures in infancy: a malignant disorder with developmental arrest. Epilepsia. 1995;36:1017-24.

De Fusco $M$, et al. The nicotinic receptor beta 2 subunit is mutant in nocturnal frontal lobe epilepsy. Nat Genet. 2000;26:275-6.

D'Gama AM, et al. Mammalian target of rapamycin pathway mutations cause hemimegalencephaly and focal cortical dysplasia. Ann Neurol. 2015;77(4):720-5.

Díaz-Otero F, et al. Autosomal dominant nocturnal frontal lobe epilepsy with a mutation in the CHRNB2 gene. Epilepsia. 2008;49(3):516-20.

Dibbens LM, et al. Mutations in DEPDC5 cause familial focal epilepsy with variable foci. Nat Genet. 2013;45:546-51.

Dobesberger J, et al. Successful surgical treatment of insular epilepsy with nocturnal hypermotor seizures. Epilepsia. 2008;49:159-62.

Fedi $\mathrm{M}$, et al. Reduced striatal D1 receptor binding in autosomal dominant nocturnal frontal lobe epilepsy. Neurology. 2008;71:795-8.

Ferri $L$, et al. A stereo EEG study in a patient with sleep-related hypermotor epilepsy due to DEPDC5 mutation. Seizure. 2017:53:51-4.

Gibbs SA, et al. Sleep- related epileptic behaviors and non-REM-related parasomnias: insights from stereo-EEG. Sleep Med Rev. 2016;25:4-20.

Harvey AS, et al. Defining the spectrum of international practice in pediatric epilepsy surgery patients. Epilepsia. 2008;49:146-55.

Heron SE, et al. Missense mutations in the sodium-gated potassium channel gene KCNT1 cause severe autosomal dominant nocturnal frontal lobe epilepsy. Nat Genet. 2012;44:1188-90.

Hildebrand MS, et al. PRIMA1 mutation: a new cause of nocturnal frontal lobe epilepsy. Ann Clin Transl Neurol. 2015;2(8):821-30.

Hirose $\mathrm{S}$, et al. A novel mutation of CHRNA4 responsible for autosomal dominant nocturnal frontal lobe epilepsy. Neurology. 1999;53(8):1749-53.

Hoda JC, et al. Human nocturnal frontal lobe epilepsy: pharmocogenomic profiles of pathogenic nicotinic acetylcholine receptor beta-subunit mutations outside the ion channel pore. Mol Pharmacol. 2008;74(2):379-91.

Ishida S, et al. Mutations of DEPDC5 cause autosomal dominant focal epilepsies. Nat Genet. 2013;45:552-5

Kim GE, et al. Human slack potassium channel mutations increase positive cooperativity between individual channels. Cell Rep. 2014;9:1661-72.

Korenke GC, et al. Nocturnal frontal lobe epilepsy caused by a mutation in the GATOR1 complex gene NPRL3. Epilepsia. 2016;57(3):e60-3.

Kurahashi H, Hirose S. Autosomal Dominant Nocturnal Frontal Lobe Epilepsy. In: Pagon RA, Adam MP, Ardinger HH, Wallace SE, Amemiya A, Bean LJH, Bird TD, Ledbetter N, Mefford HC, Smith RJH, Stephens K, editors. GeneReviews ${ }^{\circledast}$. Seattle: University of Washington Seattle; 2002. p. 19932017. Available from http://www.ncbi.nlm.nih.gov/books/NBK1169/ [Initial Posting: May 16, 2002; Last Update: March 15, 2018].

Lal D, et al. DEPDC5 mutations in genetic focal epilepsies of childhood. Ann Neurol. 2014;75:788-92

Leniger $\mathrm{T}$, et al. A new Chrna4 mutation with low penetrance in nocturnal frontal lobe epilepsy. Epilepsia. 2003;44(7):981-5.

Licchetta L, et al. Sleep-related hypermotor epilepsy: long-term outcome in a large cohort. Neurology. 2017;88(1):70-7.

Liu $\mathrm{H}$, et al. The identification of a novel mutation of nicotinic acetylcholine receptor gene CHRNB2 in a Chinese patient: its possible implication in nonfamilial nocturnal frontal lobe epilepsy. Epilepsy Res. 2011;95(1-2):94-9.

Lugaresi E, Cirignotta F. Hypnogenic paroxysmal dystonia: epileptic seizure or a new syndrome? Sleep. 1981;4(2):129-38.
Maccario M, Lustman LI. Paroxysmal nocturnal dystonia presenting as excessive daytime somnolence. Arch Neurol. 1990;47:291-4.

Marini C, Guerrini R. The role of the nicotinic acetylcholine receptors in sleeprelated epilepsy. Biochem Pharmacol. 2007:74:1308-14.

Marsan E, et al. Depdc5 knockout rat: a novel model of mTORopathy. Neurobiol Dis. 2016;89:180-9.

Martin C, et al. A recurrent mutation in DEPDC5 predisposes to focal epilepsies in the French-Canadian population. Clin Genet. 2014;86:570-4.

McLellan A, et al. Phenotypic comparison of two Scottish families with mutations in different genes causing autosomal dominant nocturnal frontal lobe epilepsy. Epilepsia. 2003;44:613-7.

Menghi V, et al. Sleep-related hypermotor epilepsy: prevalence, impact and management strategies. Nat Sci Sleep. 2018;10:317-26.

Mikati MA, et al. Quinidine in the treatment of KCNT1-positive epilepsies. Ann Neurol. 2015;78(6):995-9.

Milligan CJ, et al. KCNT1 gain of function in 2 epilepsy phenotypes is reversed by quinidine. Ann Neurol. 2014;75:581-90.

Møller RS, et al. Mutations in KCNT1 cause a spectrum of focal epilepsies. Epilepsia. 2015;56(9):e114-20.

Montagna P. Nocturnal paroxysmal dystonia and nocturnal wandering. Neurology. 1992:42:61-7.

Montagna $P$, et al. Nocturnal epileptic seizures versus the arousal parasomnias. Somnologie. 2008;12:25-37.

Montavont A, et al. Hypermotor seizures in lateral and mesial parietal epilepsy. Epilepsy Behav. 2013;28:408-12.

Nakashima M, et al. Somatic mutations in the MTOR gene cause focal cortical dysplasia type Ilb. Ann Neurol. 2015;78:375-86.

Naldi l, et al. Tobacco habits in nocturnal frontal lobe epilepsy. Epilepsy Behav. 2013;26:114-7.

Nguyen DK, et al. Revisiting the role of the insula in refractory partial epilepsy. Epilepsia. 2009;50:2599-604.

Nobili L, et al. Nocturnal frontal lobe epilepsy: intracerebral recordings of paroxysmal motor attacks with increasing complexity. Sleep. 2003;26:883-6.

Nobili $L$, et al. Sleep-related hyperkinetic seizures of temporal lobe origin. Neurology. 2004;62:482-5

Nobili $L$, et al. Surgical treatment of drug-resistant nocturnal frontal lobe epilepsy. Brain. 2007;130:561-73.

Nobili $L$, et al. Taylor's focal cortical dysplasia increases the risk of sleep-related epilepsy. Epilepsia. 2009:50:2599-604.

Ohba $\mathrm{C}$, et al. De novo KCNT1 mutations in early-onset epileptic encephalopathy. Epilepsia. 2015;56(9):e121-8.

Oldani A, et al. Autosomal dominant nocturnal frontal lobe epilepsy: a videopolysomnographic and genetic appraisal of 40 patients and delineation of the epileptic syndrome. Brain. 1998;121:205-23.

Peled R, Lavie P. Paroxysmal awakenings from sleep associated with excessive daytime somnolence: a form of nocturnal epilepsy. Neurology. 1986;36:95-8.

Phillips HA, et al. Localization of a gene for autosomal dominant nocturnal frontal lobe epilepsy to chromosome 20q13.2. Nat Genet. 1995;10:117-8.

Phillips HA, et al. A de novo mutation in sporadic nocturnal frontal lobe epilepsy. Ann Neurol. 2000;48(2):264-7.

Phillips HA, et al. CHRNB2 is the second acetylcholine receptor subunit associated with autosomal dominant nocturnal frontal lobe epilepsy. Am J Hum Genet. 2001;68(1):225-31.

Picard $F$, et al. Alteration of the in vivo nicotinic receptor density in ADNFLE patients: a PET study. Brain. 2006:129:2047-60.

Picard $F$, et al. DEPDC5 mutations in families presenting as autosomal dominant nocturnal frontal lobe epilepsy. Neurology. 2014;82:2101-6.

Proserpio $P$, et al. Insular-opercular seizures manifesting with sleep-related paroxysmal motor behaviors: a stereo-EEG study. Epilepsia. 2011;52:1781-91.

Provini F, et al. Nocturnal frontal lobe epilepsy. A clinical and polygraphic overview of 100 consecutive cases. Brain. 1999;122:1017-31.

Puligheddu $\mathrm{M}$, et al. Rationale for an adjunctive therapy with fenofibrate in pharmacoresistant nocturnal frontal lobe epilepsy. Epilepsia. 2017;58:1762-70

Rheims S, et al. Analysis of clinical patterns and underlying epileptogenic zones of hypermotor seizures. Epilepsia. 2008;49:2030-40.

Ricos $M G$, et al. Mutations in the mammalian target of rapamycin pathway regulators NPRL2 and NPRL3 cause focal epilepsy. Ann Neurol. 2016;79: 120-31.

Rizzo F, et al. Characterization of two de novo KCNT1 mutations in children with malignant migrating partial seizures in infancy. Mol Cell Neurosci. 2016;72: 54-63. 
Rozycka A, et al. Evidence for S284L mutation of the CHRNA4 in a white family with autosomal dominant nocturnal frontal lobe epilepsy. Epilepsia. 2003; 44(8):1113-7

Rubboli G, et al. Mild malformations of cortical development insleep-related hypermotor epilepsy due to KCNT1 mutations. Ann Clin Transl Neurol. 2018; 6(2):386-91.

Ryvlin P, Minotti L, Demarquay G, et al. Nocturnal hypermotor seizures, suggesting frontal lobe epilepsy, can originate in the insula. Epilepsia. 2006; 47:755-65.

Sáenz A, et al. Autosomal dominant nocturnal frontal lobe epilepsy in a Spanish family with a Ser252Phe mutation in the CHRNA4 gene. Arch Neurol. 1999; 56(8):1004-9.

Sansoni $V$, et al. A de novo mutation in an Italian sporadic patient affected by nocturnal frontal lobe epilepsy. J Sleep Res. 2012;21:352-3.

Sansoni $V$, et al. Functional characterization of a CRH missense mutation identified in an ADNFLE family. PLoS One. 2013;8(4):e61306.

Scerri T, et al. Familial cortical dysplasia type IIA caused by a germline mutation in DEPDC5. Ann Clin Transl Neurol. 2015;2(5):575-80.

Scheffer $\mathrm{IE}$, et al. Autosomal dominant frontal epilepsy misdiagnosed as sleep disorder. Lancet. 1994;343:515-7.

Scheffer $I E_{\text {, et }}$ al. Mutations in mammalian target of rapamycin regulator DEPDC5 cause focal epilepsy with brain malformations. Ann Neurol. 2014;75:782-7.

Schwalen S, Jorg J. Day-time fatigue in frontal lobe epilepsy with primarily sleeprelated seizures. A case report. Nervenarzt. 1998;69:166-70.

Shimada S, et al. A novel KCNT1 mutation in a Japanese patient with epilepsy of infancy with migrating focal seizures. Hum Genome Var. 2014;1:14027.

Steinlein OK. Genetic heterogeneity in familial nocturnal frontal lobe epilepsy. Prog Brain Res. 2014;213:1-15.

Steinlein OK, et al. A missense mutation in the neuronal nicotinic acetylcholine receptor a4 subunit is associated with autosomal dominant nocturnal frontal lobe epilepsy. Nat Genet. 1995;11:201-3.

Steinlein OK, et al. An insertion mutation of the CHRNA4 gene in a family with autosomal dominant nocturnal frontal lobe epilepsy. Hum Mol Genet. 1997; 6(6):943-7.

Steinlein OK, et al. Independent occurrence of the CHRNA4 Ser248Phe mutation in a Norwegian family with nocturnal frontal lobe epilepsy. Epilepsia. 2000; 41(5):529-35.

Steinlein OK, et al. Mutations in familial nocturnal frontal lobe epilepsy might be associated with distinct neurological phenotypes. Seizure. 2012;21:118-23.

Terzaghi $\mathrm{M}$, et al. Coupling of minor motor events and epileptiform discharges with arousal fluctuations in NFLE. Epilepsia. 2008;49:670-6.

Tinuper P, Bisulli F. From nocturnal frontal lobe epilepsy to sleep-related Hypermotor epilepsy: a 35-year diagnostic challenge. Seizure. 2017:44:87-92.

Tinuper $\mathrm{P}$, et al. Nocturnal paroxysmal dystonia with short-lasting attacks: three cases with evidence for an epileptic frontal lobe origin of seizures. Epilepsia. 1990;31(5):549-56.

Tinuper $\mathrm{P}$, et al. Definition and diagnostic criteria of sleep-related hypermotor epilepsy. Neurology. 2016:86:1834-42.

van Kranenburg M, et al. Preliminary functional assessment and classification of DEPDC5 variants associated with focal epilepsy. Hum Mutat. 2015;36(2):200-9.

Vaugier $L$, et al. Neural networks underlying hyperkinetic seizures of "temporal lobe" origin. Epilepsy Res. 2009;86:200-8.

Vignatelli $L$, et al. Excessive daytime sleepiness and subjective sleep quality in patients with nocturnal frontal lobe epilepsy: a case-control study. Epilepsia. 2006;47(suppl 5):73-7

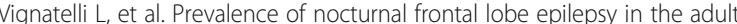
population of Bologna and Modena, Emilia-Romagna region, Italy. Sleep. 2015;38:479-85.

Vignatelli $L$, et al. Prevalence of nocturnal frontal lobe epilepsy in the adult population of Bologna and Modena, Emilia-Romagna region. Italy Sleep. 2017;1(2):40.

Wang MY, et al. A novel mutation of the nicotinic acetylcholine receptor gene CHRNA4 in a Chinese patient with non-familial nocturnal frontal lobe epilepsy. Epilepsy Res. 2014;108(10):1927-31.

Waterman $\mathrm{K}$, et al. An epileptic syndrome caused by mesial frontal lobe seizure foci. Neurology. 1987;37(4):577-82.

Wiebe $\mathrm{S}$, et al. A randomized, controlled trial of surgery for temporal-lobe epilepsy. N Engl J Med. 2001;345:311-8.

Williamson PD, et al. Complex partial seizures of frontal lobe origin. Ann Neurol. 1985;18(4):497-504
Willoughby JO, et al. Nicotine as an antiepileptic agent in ADNFLE: an N-of-one study. Epilepsia. 2003:44(9):1238-40.

Zucconi $\mathrm{M}$, et al. The macrostructure and microstructure of sleep in patients with autosomal dominant nocturnal frontal lobe epilepsy. J Clin Neurophysiol. 2000;17:77-86.

\section{Publisher's Note}

Springer Nature remains neutral with regard to jurisdictional claims in published maps and institutional affiliations.
Ready to submit your research? Choose BMC and benefit from:

- fast, convenient online submission

- thorough peer review by experienced researchers in your field

- rapid publication on acceptance

- support for research data, including large and complex data types

- gold Open Access which fosters wider collaboration and increased citations

- maximum visibility for your research: over $100 \mathrm{M}$ website views per year

At BMC, research is always in progress.

Learn more biomedcentral.com/submissions 